\title{
Géoendoscopie : application \\ à la reconnaissance \\ et au diagnostic en site urbain
}

\section{P. BREUL \\ R. GOURVES}

Lermes-C/U/S/T Univ.

Blaise-Pascal-Clermont II

Campus universitaire

des Cézeaux

24, av. des Landais, BP 206

63174 Aubière Cedex

Breul@lermes.univ-bpcler-

mont.fr

\section{y. HADDANI}

Sol Solution

ZAC des Portes-de-Riom 63200 Riom
La géoendoscopie, technique mise au point il y a quelques années, est basée sur l'utilisation de l'endoscopie et de l'analyse d'images. Utilisée dans des domaines d'application très variés, elle continue à être développée tant sur le plan technologique que sur le plan de l'analyse pour répondre à des problématiques particulières. Cet article présente justement I'application de la géoendoscopie au diagnostic et à la reconnaissance en site urbain où ses atouts (essai in situ, faible encombrement, rapicité de réalisation et automatisation) permettent de bien s'adapter à ce contexte (site d'accès difficile et limité, variabilité importante des milieux sondés, présence de réseaux....). La première partie de I'article présente globalement le principe technique et l'exploitation usuelle des images. Dans la seconde partie, nous présentons les possibilités offertes par la géoendoscopie pour le diagnostic de maçonnerie et le diagnostic et le contrôle d'ouvrages enterrés.

Mots-clés : endoscopie, analyse d'images, diagnostic, site urbain.

\section{Geoendoscopy : application to soil investigation and diagnostic in urban area}

Geoendoscopy is based on the use of endoscopy and image analysis. Used in a wide range of application fields, this technique keeps developping (technology and image analysis) to solve some particular issues. This paper precisely deals with using geoendoscopy in urban area. The advantages of this technique (in field and light test, automatic analysis and quick execution) are well aclapted to this environment (limited access. wide variability of tested area, network presence...). The first part describes the global investigation method and the usual image exploitation. We present in the second part the possibilities offered by geoendoscopy for stru cture diagnosis and burried structures control.

Key words : endoscopy, image analysis, diagnostic, urban area. 


\section{Contexte}

Les sites urbains sont par nature des sites fortement remaniés, hétérogènes, composés de remblais récents ou anciens aux caractéristiques complexes (matériaux naturels, déchets, matériaux de démolition...). Un grand nombre d'ouvrages d'infrastructure ont été réalisés il y a plus de trente ans. Ces ouvrages nécessitent un diagnostic régulier afin de quantifier leur état et dans certains cas un entretien ou une réhabilitation afin de les régénérer et d'augmenter ainsi leur durée de vie. Du fait du contexte (réseaux, endroit d'accès difficile...) l'établissement d'un diagnostic et la reconnaissance par des méthodes classiques est souvent difficile. De plus, du fait de la variabilité extrême de ces milieux, il est nécessaire d'avoir à sa disposition des outils permettant de réaliser des sondages rapides et peu onéreux pour pouvoir les multiplier. C'est pourquoi l'utilisation de la géoendoscopie, technique portable utilisant I'endoscopie (appareil optique de faible encombrement) et l'analyse d'images peuvent être un outil intéressant pour ce domaine d'application.

Cet article présente la technique de la géoendoscopie associée à des forages mécaniques préalables (forages et/ou essais pénétrométriques notamment) de faible diamètre et à faible profondeur (de l'ordre de quelques mètres). A partir du couplage des informations mécaniques et morphologiques, différentes caractéristiques peuvent être analysées. La rapidité d'exécution des sondages et leur analyse automatique permettent en outre l'application des méthodes géostatistiques.

L'article propose dans un premier temps de présenter la technique géoendoscopique et le traitement effectué puis tente de démontrer son intérêt en site urbain, notamment, sur quelques exemples d'application réels.

\section{Géoendoscopie: principe technique et exploitation}

\section{Principe technique}

Si l'utilisation de l'endoscopie dans le milieu médical est devenue relativement familière, dans l'industrie, bien qu'elle soit répandue et couvre de nombreux champs d'application (notamment en mécanique, aéronautique et nucléaire), elle reste méconnue dans le domaine du génie civil. De plus, lorsqu'elle est utilisée c'est le plus souvent de manière qualitative (observation d'un milieu d'accès difficile). Quelques développements sont réalisés à T'heure actuelle dans d'autres pays (Lieberman et Anderson, 1999) (Rashke, 1996) pour tenter d'obtenir des informations quantitatives sur les caractéristiques du milieu observé. Depuis quelques années, nous avons développé cette technique dans le domaine des milieux granulaires en particulier et des géomatériaux, en général.

Parmi les différentes familles d'endoscopes existantes (endoscope rigide, vidéoendoscope, fibroscope), notre choix s'est orienté vers les vidéoendoscopes. Ces appareils sont des endoscopes souples, de longueur libre (d'un à plusieurs dizaines de mètres), comportant à leur extrémité un capteur CCD et un système d'éclairage propre (fibres optiques, LED, ampoule). Leur miniaturisation et leur souplesse d'utilisation (grande longueur, emploi en cavités non obligatoirement rectiligne, éclairage adaptable et puissant, grande résolution d'images) ont été des arguments forts pour définir notre choix.

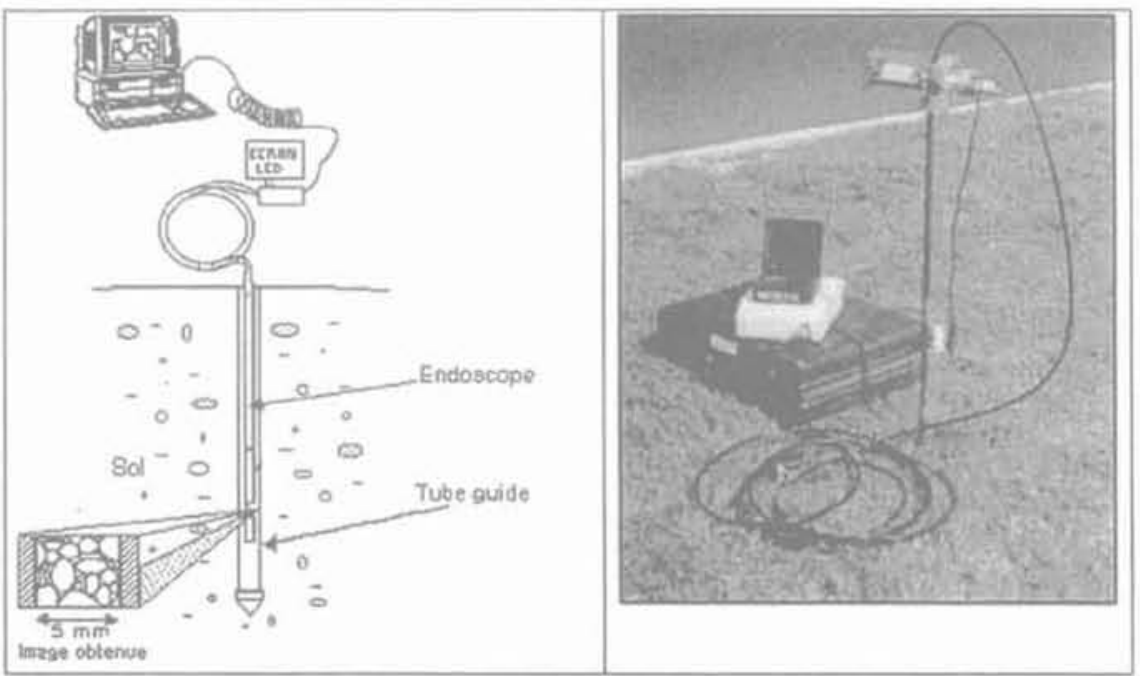

FG. 1 Schéma de principe et vue in situ du matériel de géoendoscopie. Apparatus principle and in situ presentation. 
Pour répondre aux contraintes particulières de nos domaines d'application (milieux granulaires, secs ou humides, matériaux effondrables, abrasifs...), plusieurs adaptations et des prototypes ont été réalisés, notamment pour veiller à obtenir une bonne qualité d'éclairage et de prise d'images (netteté, profondeur de champ, grossissement).

La technique mise en place actuellement s'appuie sur l'utilisation d'un vidéoendoscope de diamètre $8,6 \mathrm{~mm}$, éclairé par des diodes électroluminescentes (LED) apportant une lumière homogène sur l'ensemble de la surface observée. Un potentiomètre permet de régler l'éclairage en puissance. Les images sont acquises par l'intermédiaire d'un capteur CCD (Charged Coupled Device) de $1 / 6$ de pouce haute résolution (470000 pixels). La profondeur de champ varie de $5 \mathrm{~mm}$ à $25 \mathrm{~mm}$.

L'essai endoscopique permet l'enregistrement en continu (par magnétoscope DV ou sur ordinateur) d'images de taille $5 \times 5 \mathrm{~mm}$ avec une résolution de $10 \mu \mathrm{m} /$ pixel.

La technique de réalisation d'essai (cf. Fig. 1) consiste à introduire l'endoscope dans un tubage placé dans la cavité préalablement réalisée par un essai mécanique. Le tube guide qui doit être en contact avec le terrain, est percé de fenêtres dont la dimension de l'ouverture est connue très précisément ( $5 \mathrm{~mm}$ ). Le rôle de ce tube guide est triple : tout d'abord protéger l'endoscope lors de l'auscultation, maintenir celui-ci le plus stable possible lors de la prise d'images et enfin fournir à l'opérateur le grossissement des images. En effet, l'une des difficultés d'emploi de l'endoscopie est de connaître le grossissement auquel on travaille puisque ce grossissement évolue notablement en fonction de la distance objet observé/endoscope.

Suite à la mise en place du tube guide, l'endoscope est placé à son tour à l'intérieur des tubes et descendu jusqu'au fond de la cavité. Un film continu est enregistré pendant le déplacement del'endoscope d'une extrémité à l'autre de la cavité, puis des images sont extraites de ce film à intervalles réguliers.

D'un point de vue pratique cette technique permet aujourd'hui l'auscultation de forage de diamètre mini$\mathrm{mal}$ : $12 \mathrm{~mm}$, de longueur 6 à $7 \mathrm{~m}$ et nécessite environ une demi-heure pour réaliser un essai complet (installation, réalisation, repli du matériel).

Des exemples d'images obtenues dans différents domaines d'application (milieux granulaires, maçonneries, archéologie) sont présentés figure 2.

\section{2}

\section{Exploitation}

Une fois les images acquises, le travail consiste à extraire des informations pertinentes d'un point de vue géotechnique et à mettre au point des analyses automatiques permettant de donner des valeurs fiables des caractéristiques étudiées.

Avant toute analyse préalable, il est nécessaire de traiter les images, afin d'éliminer tout ou partie des reflets parasites éventuels ainsi que les problèmes d'hétérogénéité d'éclairage. Cette étape est réalisée par l'utilisation d'outils de traitement d'images basés sur la théorie du traitement du signal et de la morphologie mathématique (filtrage, chapeau haut de forme, table de conversion...) (Cocquerez et Philipp, 1995), (Coster et Chermant, 1989).

A partir des images traitées, nous nous sommes intéressés dans un premier temps à l'analyse de texture qui, comme l'ont montré Rashke (1996) et Breul et al. (1999), permet une caractérisation partielle des matériaux. Cette analyse a pour but de dissocier et de carac-

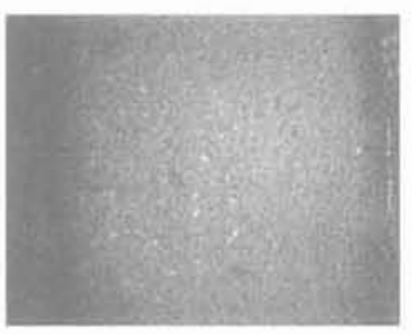

a

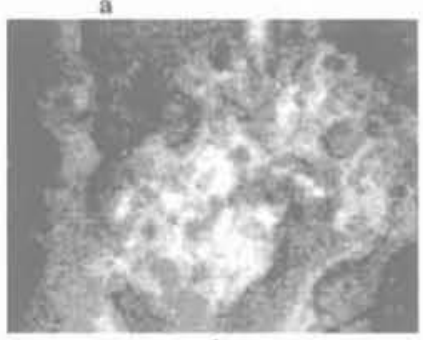

d

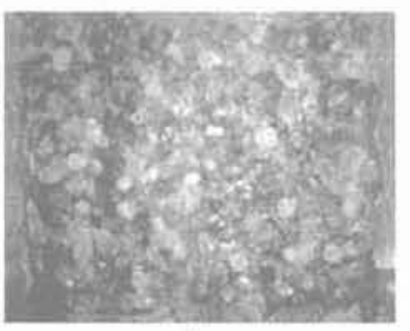

b

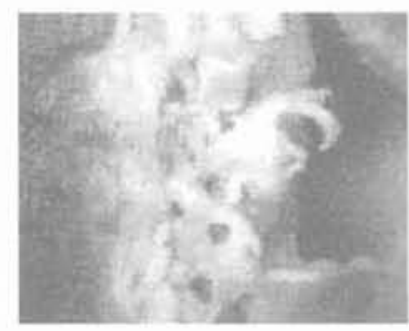

c

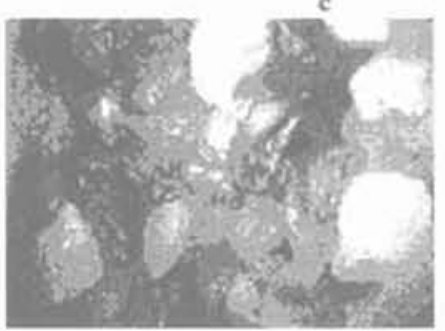

e

FIG. 2 Exemple d'images obtenues $(\mathrm{a}$ : limon ; $\mathrm{b}$ : sable fin ; $\mathbf{c}$ : ossements ; d : maçonnerie de meulière altérée ; e : sable grossier).

Images obtained with geoendoscopy (a : silt ; b : fine sand; $\mathrm{c}$ : bones ; d : damaged structure ; e : coarse sand). 
tériser les matériaux fins ou très fins (matériaux de type A1 à 4 et B5 et B6 au sens de la classification GTR du Setra (1994)) sur lesquels aucune analyse granulométrique n'est envisageable du fait de l'impossibilité d'individualiser les grains sur les images. En effet, du fait du pourcentage de particules fines (de manière générale, les particules de diamètre inférieur à $80 \mu \mathrm{m}$ ) et avec le grossissement utilisé lors de l'observation, les images ont un aspect plus ou moins « lisse $»$. L'étude de la texture est basée sur l'analyse spectrale de l'image et l'étude du module de la transformée de Fourier de l'image (plus particulièrement sur l'étude du moment normalisé d'ordre 3 (M3) du logarithme normalisé du spectre de puissance) ainsi que sur une analyse statistique de l'histogramme en niveau de gris (coefficient de variation et écart type).

Sur les images de matériaux possédant moins de $12 \%$ de particules inférieures à $80 \mu \mathrm{m}$, nous avons mis en place des procédures automatiques d'analyse granulométrique. Ces procédures sont basées sur l'emploi de méthodes par ouverture morphologique et de méthodes d'analyse des traversées (segment interceptant les particules dans l'image) (Breul, 1999). Cette analyse est réalisable à l'heure actuelle sur des matériaux sableux ne possédant pas de particules de diamètre supérieur à $5 \mathrm{~mm}$.

La couleur et la forme des particules sont les deux autres caractéristiques qui ont été étudiées. La couleur permet d'obtenir une reconnaissance stratigraphique automatique du milieu sondé. Cette caractéristique s'appuie sur les critères de teinte de saturation et d'intensité globale de l'image. La forme des particules s'appuie sur l'étude de morceaux de contours extraits à partir des images et de leur analyse automatique pour dissocier les matériaux roulés des matériaux concassés.
A partir de l'analyse des images, des graphiques donnant l'évolution des différentes caractéristiques précitées en fonction de la profondeur du sondage sont réalisés afin d'effectuer une analyse couplée des résultats mécaniques et morphologiques (cf. Fig. 3).

\section{3}

\section{Application au diagnostic d'ouvrages enterrés}

Les ouvrages enterrés (tunnels, galeries techniques, réseaux d'assainissement...) subissent des agressions très fortes dues aux régimes hydrogéologiques des terrains environnant l'ouvrage ainsi que des surcharges dynamiques liẻes au développement du trafic urbain. Ces dernières augmentent considérablement le vieillissement et la dégradation de ces ouvrages. Ce patrimoine en majorité construit depuis plus de trente ans accuse un certain nombre de désordres pouvant modifier ou empêcher les fonctions pour lesquels l'ouvrage est destiné. Les causes de ces désordres sont multiples (facteurs intrinsèques, présence d'eau, effluents véhiculés par l'ouvrage, terrain encaissant). La gestion de ce type d'ouvrage implique donc la nécessité de les entretenir et de les réhabiliter pour conserver ou rétablir leurs fonctions d'origine voire pour les améliorer afin de tenir compte des nouvelles normes techniques, de sécurité ou d'hygiène sanitaire.

L'objectif du diagnostic de tels ouvrages est de déterminer l'état de la structure, d'observer l'interface sol/structure (le contact) et de caractériser le milieu encaissant (état de serrage, injectabilité, cavités...). De nombreuses méthodes de diagnostic existent (inspec-

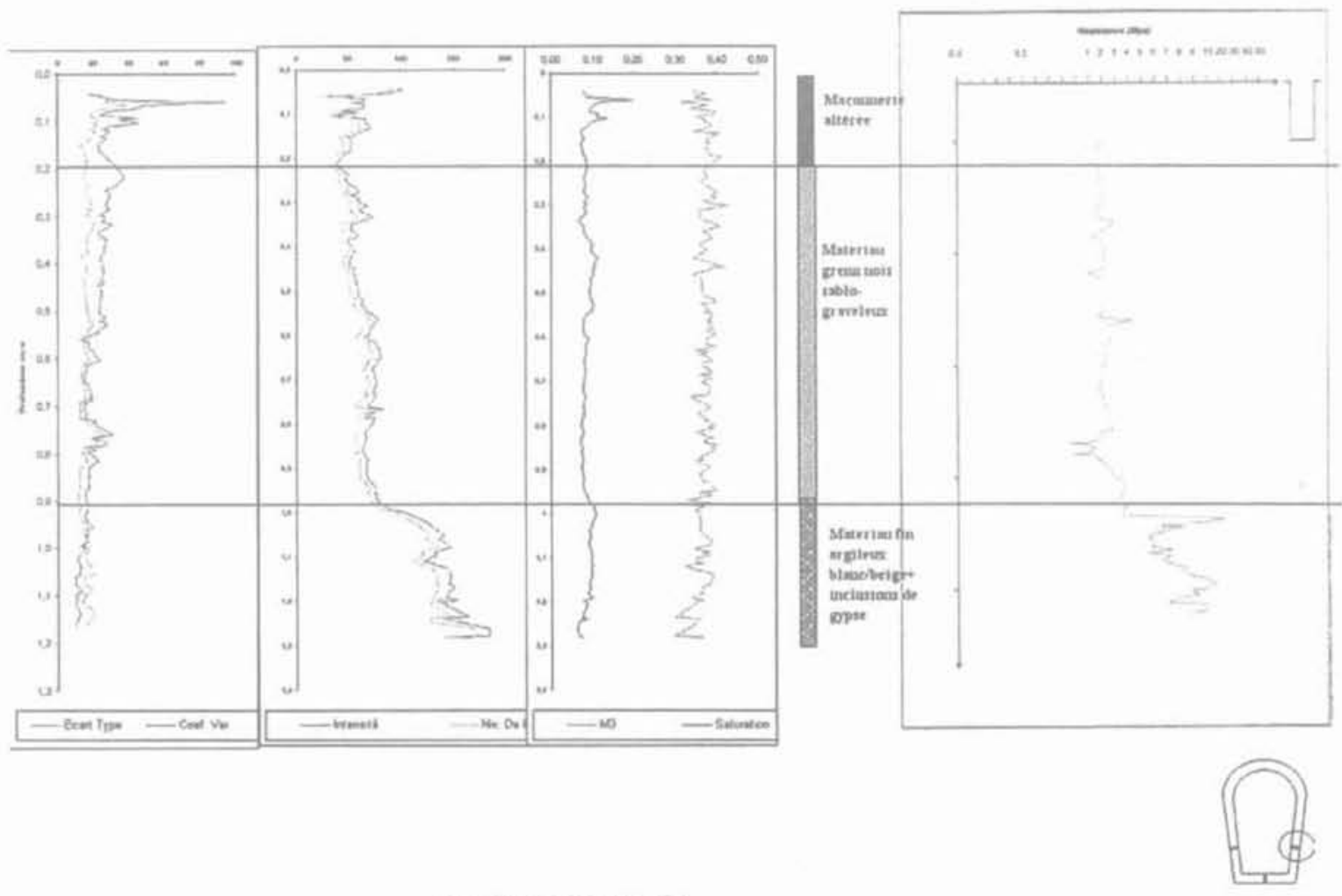

ANALYSE ENDOSCOPIQUE -

FIG. 3 Exemple de résultats d'analyse endoscopique et pénétrométrique effectué dans le même sondage. Results of geoendoscopy and light dynamic penetrometric test realised in the same boring. 
tion visuelle; méthodes géophysiques : radar, électrique... ; système MAC - mécanique d'auscultation des conduits - essai basé sur la sollicitation des parois d'un ouvrage par vérinage ; sondages destructifs ; essais d'eau) mais la géo-endoscopie couplée à des essais de forages mécaniques peut permettre de caractériser l'état général de l'ouvrage et de chacune de ses parties (structure, contact, encaissant) individuellement ou d'améliorer notablement les analyses effectuées à partir d'essais réalisés avec des méthodes globales.

Nous allons présenter son intérêt sur un exemple de diagnostic de maçonnerie, ainsi que sur la caractérisation complète d'une canalisation visitable (structure, contact et encaissant).

\section{1}

\section{Diagnostic de maçonnerie}

\section{(3) \\ Position du problème}

Les méthodes de diagnostic de maconnerie classiquement utilisées consistent essentiellement en des carottages et des essais Lugeon. L'analyse de la carotte permet de déterminer la nature des matériaux utilisés ainsí que l'épaisseur de la paroi, l'essai Lugeon permet de quantifier le débit de fuite donc l'état de fissuration de la structure.

L'utilisation de la géoendoscopie dans ce cas de figure permet d'obtenir les mêmes renseignements que le carottage et l'essai Lugeon (épaisseur, nature des matériaux, et état général de la maçonnerie) mais dans un forage plus étroit (de l'ordre de $1,5 \mathrm{~cm}$ ) donc moins traumatisant et de manière automatique et rapide (technique portable et analyses automatiques).

L'étude présentée ci-après a été réalisée dans une galerie technique sur Paris. Lobjectif était d'évaluer la possibilité de quantifier l'état des maçonneries au moyen de la géo-endoscopie (calcul du RQD, Rock Quality Designation, quantification du degré de fracturation des roches. Le RQD désigne le pourcentage de morceaux de carottes sains de longueur supérieure à $10 \mathrm{~cm}$ ) ainsi que la nature et la position des pathologies (présence de fissures, oxydation de la meulière, matériaux désagrégés...).

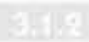

\section{Le protocole expérimental}

Pour mettre en place le diagnostic de maçonnerie par géoendoscopie, nous avons dans un premier

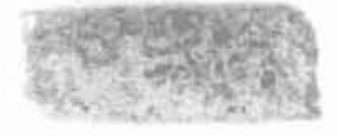

a

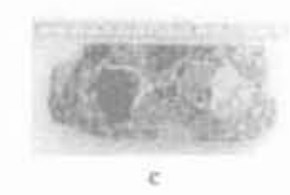

FG. 4 Exemple de carottes étudiées (a : meulière dégradée ; b : calcaire ; : maçonnerie saine).

Core examples studied (a : damaged structure b; limestone : c: sound structure). temps, étudié les caractéristicques endoscopiques d'un échantillon de carottes issues de campagnes de diagnostic antérieures afin de réaliser un paramètrage des différents matériaux (Fig. 4) et déterminer les seuils discriminants vis-à-vis des défauts (Haddani, 2001).

Les carottes concernaient trois types de matériaux:

- maçonneries béton à différents états de dégradation (saine, dégradée, fine, grossière) :

- maçonneries de meulière (saine ou oxydée) ;

- matériaux naturels (calcaire, argile).

L'étude de paramétrage consiste à établir des critères permettant de reconnaître automatiquement les différents matériaux susceptibles d'être rencontrés dans une paroi d'ouvrage enterré ainsi que les défauts de structure existants (fissure, vide...) par analyse d'images.

Les images ont été prises sur quatre génératrices de chaque carotte à $90^{\circ}$ les unes des autres. A partir de ces images, les paramètres statistiques, de texture ainsi que de couleur ont été extraits.

Les résultats obtenus nous ont permis de déterminer pour chaque caractéristique de couleur et de texture et chaque matériau des plages de valeurs où la probabilité de présence est maximale (Tableau I). L'analyse de ces valeurs met en évidence la possibilité de distinguer certains matériaux à partir de ces paramètres. En revanche, la recherche de nouveaux paramètres (de texture notamment) sera nécessaire pour permettre de mieux dissocier des matériaux dont les caractéristiques actuelles sont très proches.

A partir de ces données nous avons établi les seuils des paramètres permettant de caractériser les différents matériaux et plus particulièrement les fissures ou trous (Tableau II) dans la maçonnerie, les meulières dégradées (oxydées) ou les maçonneries dégradées (manque de liant ou liant détérioré) (Fig. 5).

TABLEAU Extrait de tableau récapitulatif des paramètres relatifs aux différents matériaux étudiés (intervalle cle confiance à $95 \%$ ). Sample of image parameters values obtained on different materials (confidence interval at $95 \%$ ).

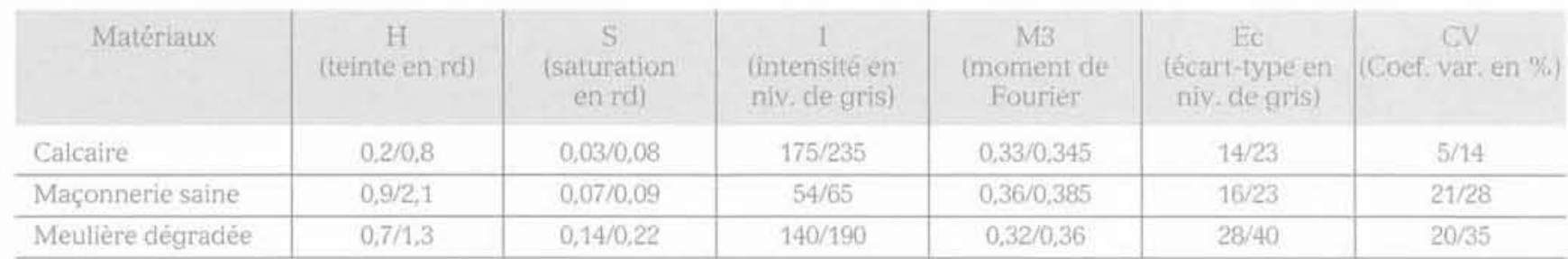


TAaLEAUII Les seuils retenus pour les fissures et les trous. Threshold values for failures and hole identification.

\begin{tabular}{c|c|c|c|c|c|c} 
& Intensité & Saturation & Teinte & Ecart-type & Coef. Var: & Moment M3 \\
\hline Fissures et trous & $\mathrm{I}<60$ & $\mathrm{~S}>0,15$ & Pas d'influence & $\mathrm{E}<25$ & $\mathrm{CV}>40$ & $\mathrm{M} 3>0,40$ \\
\hline
\end{tabular}

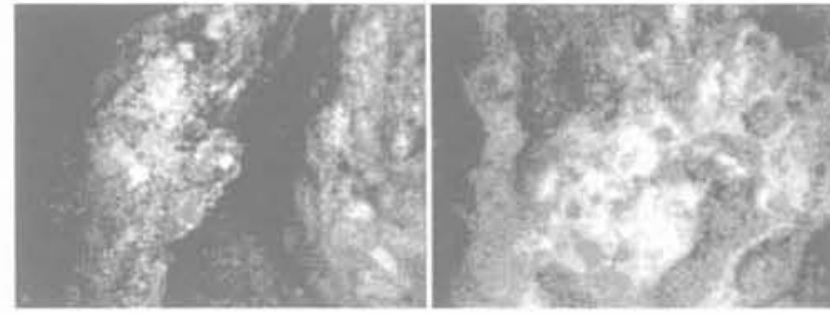

FIG.5 Image de fissure et de meulière dégradée. Failure and damaged structure image.

\section{and}

\section{Essais de validation}

Pour tester les critères de reconnaissance de matériau établis lors de l'étude de calage, nous avons appliqué les seuils mis en place sur des carottes issues d'une étude antérieure.
Au total 20 carottes ont été analysées avec des états de dégradation variables (carottes saines à carottes complètement désagrégées).

Pour chaque carotte, les résultats de l'analyse endoscopique sont présentés sous la forme de graphiques donnant l'évolution des différents paramètres pris en compte en fonction de la profondeur (Fig. 6).

La comparaison des résultats trouvés par analyse géoendoscopique avec les résultats obtenus par les méthodes classiques est concordante dans plus de $85 \%$ des cas. Ce résultat a permis de valider la robustesse des seuils choisis.

La méthode a ensuite été testée in situ à l'intérieur de carottages et de forages destructifs réalisés dans la maçonnerie d'une galerie technique. Le taux de caractérisation correcte de la maçonnerie (après comparaison avec une inspection visuelle des carottes et des résultats d'essai Lugeon) à l'intérieur des carottages reste supérieur à $85 \%$. A l'intérieur de forages destructifs, l'obtention de tels objectifs nécessite d'effectuer un nettoyage préalable (à l'eau ou air sous pression) du forage. En effet, du fait du mode de forage (à sec), l'état apparent de la maçonnerie est modifié par l'accumulation de poussière sur les parois.

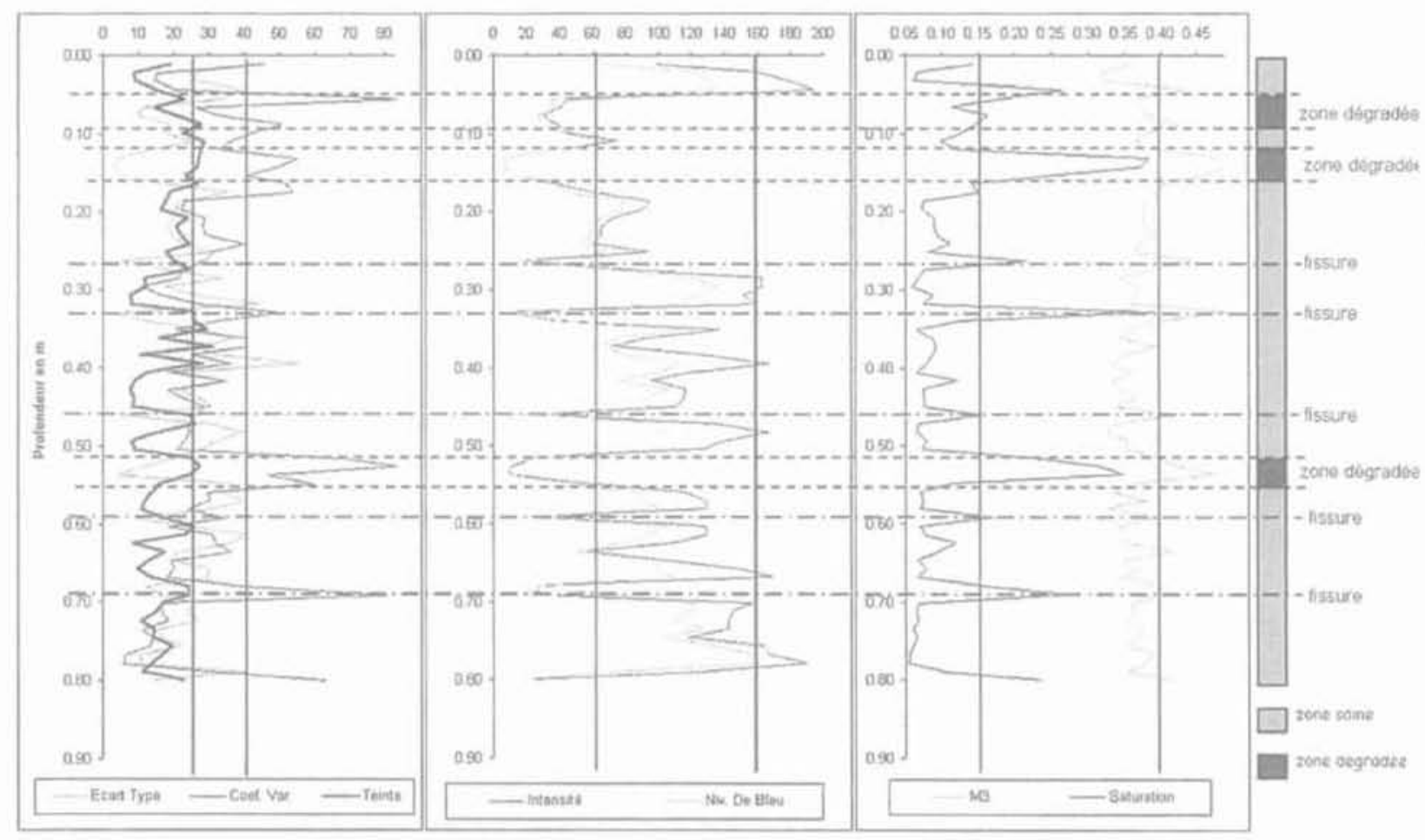

ANALYSE ENDOSCOPIQUE

CAROTTE 8 Maçonnerie cassée. CT argileux

\begin{tabular}{|l|r|}
\hline Nombre de fissures & 3 \\
\hline Nombre de zones dégradéses & 3 \\
\hline Pourcentage de dégradation & $10 \%<<30 \%$ \\
\hline Etat de la carotte & Moyen \\
\hline
\end{tabular}

FG.6 Document résultat du diagnostic de maçonnerie par endoscopie. Structure diagnosis result by using geoendoscopy. 

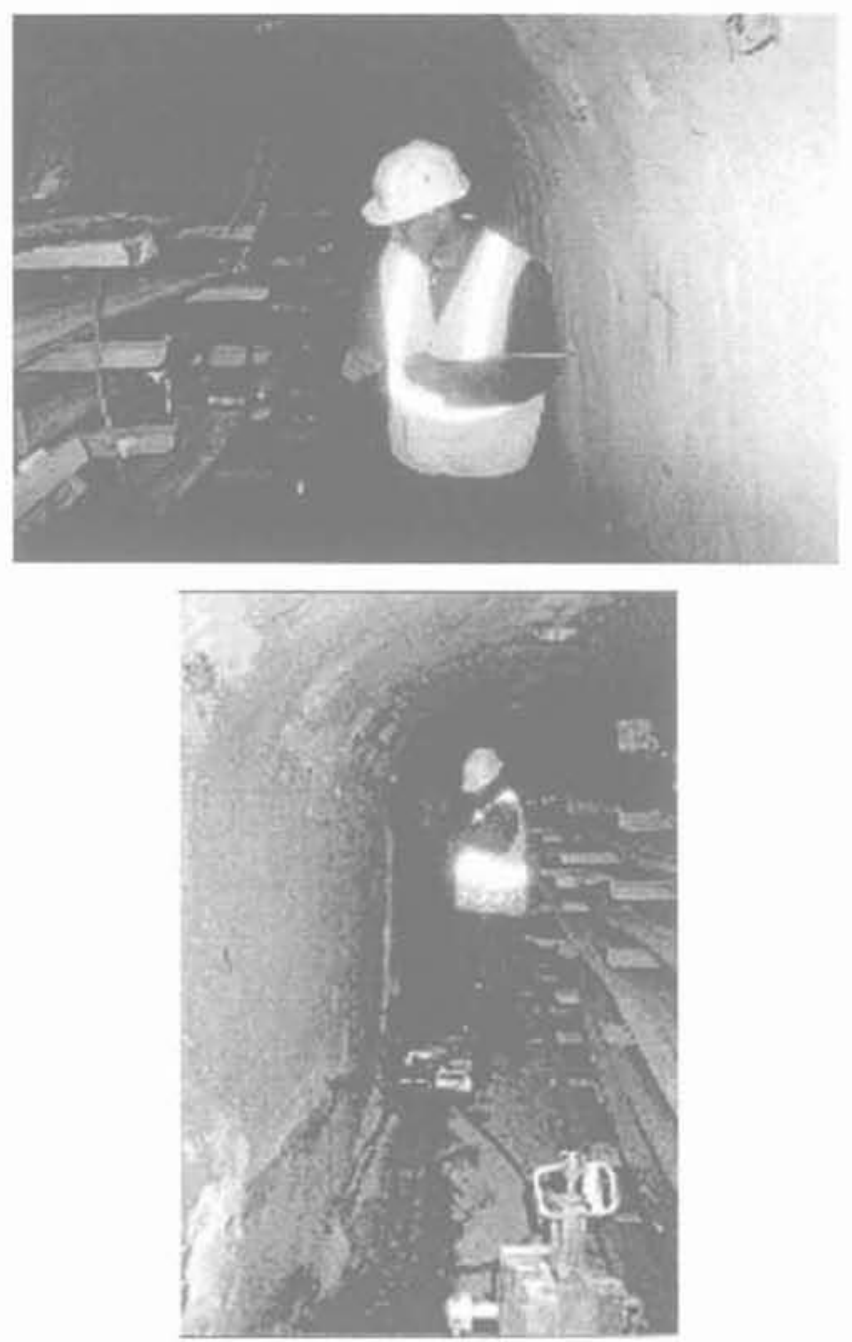

FIG. 7 Réalisation de sondages dans une galerie technique.

Test realisation in a technical buried structure. évidence la présence éventuelle de vides entre la maçonnerie et l'encaissant mais aussi à décrire l'état de l'encaissant juste derrière la structure. Ces caractéristiques sont fournies par le couplage du pénétrogramme et des images enregistrées (Fig. 3).

Pour tester cette méthode, nous avons réalisé une campagne d'essais dans un tronçon de canalisation visitable.

- Description de l'ouvrage

L'ouvrage dans lequel les sondages ont été effectués est un ovoïde de type 180/100 (Fig. 8) sur une longueur de 223 m fonctionnant en unitaire. La date de construction de cet ouvrage est estimée au début du $\mathrm{XX}$ ' siècle.

L'ouvrage a été réalisé en maçonnerie de meulière hourdée au béton 0/10 sur l'ensemble du linéaire. Il est situé sous la chaussée à une profondeur allant de $70 \mathrm{~cm}$ à $105 \mathrm{~cm}$. Ce troncon comprend cinq regards de visite. L'épaisseur de la maçonnerie est de l'ordre de $20 \mathrm{~cm}$ et est particulièrement en mauvais état (aspect visuel).

\section{- Contexte hydrogéologique}

D'un point de vue géologique, le tronçon repose sur un sol d'assise constitué de marnes infra-gypseuses, l'encaissant est composé de ce même matériau. Le risque géologique principal de cette formation est lié aux phénomènes de dissolutions pouvant se manifester sous forme d'affaissements (remontées de fontis). De plus, la présence de gypse résiduel et de produits de substitution dans les couches les plus marneuses crée des hétérogénéités présentant des risques de tassement différentiel.

Quant au régime hydrogéologique, le réseau est hors nappe, cependant des circulations d'eau confuses sont susceptibles d'exister au sein des marnes infragypseuses.

L'objectif de cette étude est triple:

- déceler la présence éventuelle de cavités ou de zone de fontis en radier ou sur les flancs du collecteur ;

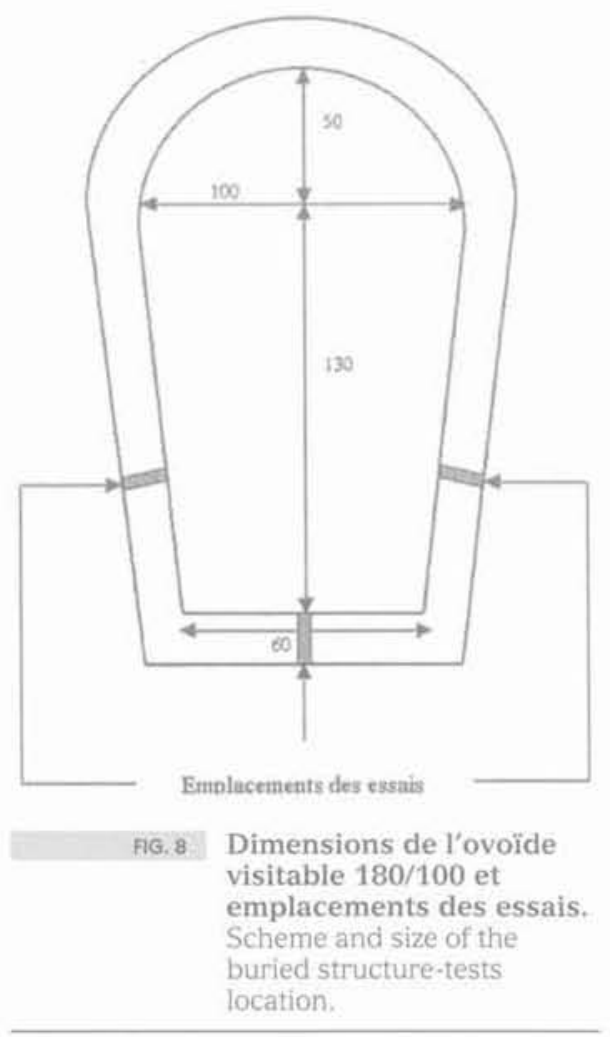


- diagnostiquer l'état de la structure et de l'encaissant, ainsi que la présence éventuelle de vides au niveau du contact encaissant/structure ;

- voir dans quelle mesure l'encaissant est injectable.

\section{lopere}

\section{Le déroulement des essais}

Les essais Panda/endoscope ont été réalisés dans les piédroits et dans le radier (Figs. 8 et 9). Préalablement à l'essai de pénétration, un forage destructif a été effectué dans la maçonnerie.

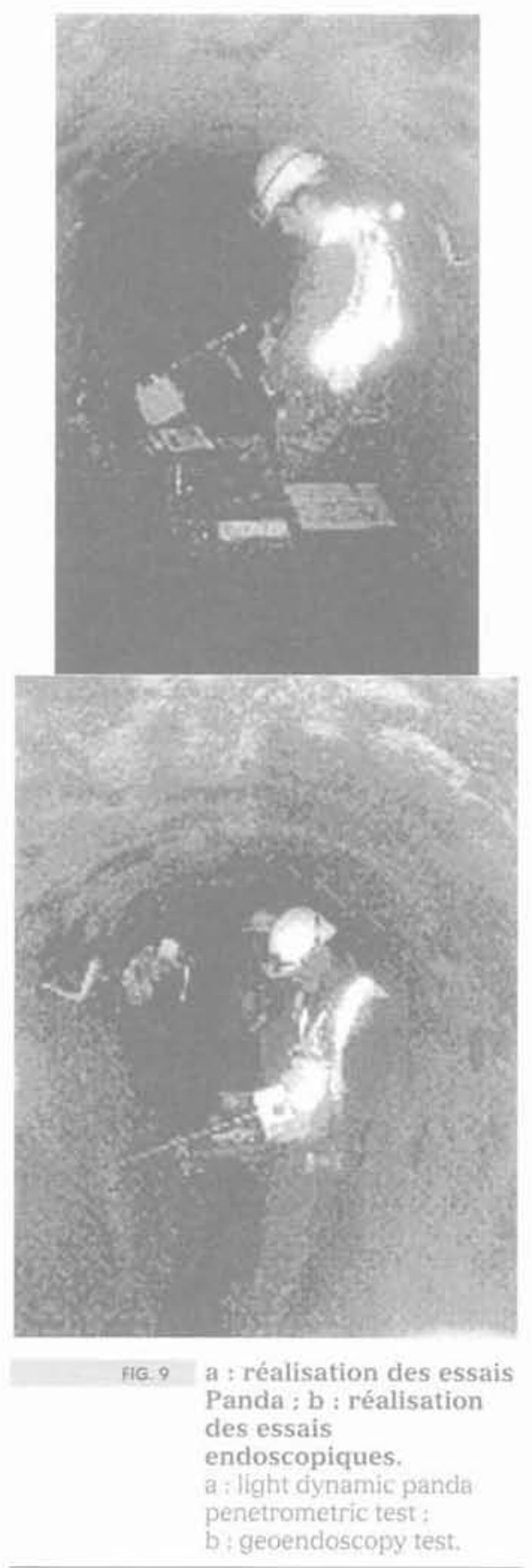

Le couplage des informations mécaniques et endoscopiques permet de détecter les couches rencontrées et de caractériser les matériaux (Fig. 3).

L'analyse des essais a permis de mettre en évidence le mauvais état général de la maçonnerie et plus particulièrement sur la première partie de l'ouvrage. Celle-ci est désagrégée par endroit (en radier notamment) et souvent altérée (liant désagrégé, présence de fissures).

Au niveau du contact structure/encaissant, plusieurs essais ont révélé la présence de vides pouvant atteindre plusieurs millimètres à cet endroit.

Concernant l'encaissant, plusieurs types de matériaux ont été mis en évidence :

- des matériaux de remblais (sables, matériau sablograveleux, matériaux fin argileux) :

- des marnes infra-gypseuses :

- du gypse sain ou altéré ;

- du sol pollué par des matières organiques provenant des effluents et sol tourbeux.

Sur ce type d'étude, la légèreté et la maniabilité de l'endoscope et du pénétromètre Panda ont été largement appréciées puisque le nombre d'essais réalisés a été multiplié par 4 par rapport à une méthode classique par carottage.

\section{4}

\section{Conclusion et perspectives}

Cet article a tenté de montrer l'intérêt de la géoendoscopie notamment pour les travaux en site urbain. Comme nous l'avons vu, cette technique permet une caractérisation des géomatériaux par analyse d'images de manière automatique. Couplée à un essai mécanique elle permet d'obtenir une caractérisation relativement complète (caractéristiques physiques et état de serrage) de l'ensembles des parties d'un ouvrage (structure, contact et encaissant). Des études complémentaires (recherche de nouveaux paramètres de texture notamment) seront nécessaires pour permettre de mieux dissocier des matériaux dont les caractéristiques sont très proches.

Le faible encombrement de cette technique et la rapidité d'exécution des sondages permettent de rẻaliser un nombre élevé d'essais dans des endroits d'accès difficiles et sur des ouvrages en service.

D'autres applications en site urbain ont été réalisées: contrôle de travaux d'injection (identifier la présence d'injection et sa répartition), diagnostic et expertise sous dallage ou chaussée (recherche des causes de pathologie de ces ouvrages), reconnaissance archéologique (recherche de l'épaisseur du milieu anthropique; caractérisation de différentes couches).

Nous remercions le Réseau génie civil et urbain et le ministère charcié de la Recherche et de la Technologie pour leur participation et leur aide au développement de ce projet.
} 
Breul P. - Caractérisation endoscopique des milieux granulaires couplée à lessai de pénétration. Thèse de docteur-ingénieur de l'université de Clermont-Ferrand. 1999, 280 pages.

Breul P., Gourvès R., Boissier D. - « In situ granular materials characterisation using endoscopy 2. Int. Symp. on imaging applications in geology. Geovision 99, 1999, p. 29-32

Cocquerez J.P. Philipp S. - Analyse d'images; filtrage et segmentation. Ed. Masson. 1995
Coster M., Chermant J.L. - Précis d'analyse d'images. Ed. CNRS, 1989.

Gourvès R. - Le PANDA ; pénétromètre dynamique léger à énergie variable pour la reconnaissance des sols. Rapport interne Laboratoire LERMES, univer sité Blaise-Pascal, Clermont-Ferrand, 1991.

Haddani Y. - $\alpha$ Développement de la géoendoscopie aux sols saturés et au diagnostic d'ouvrages enterrés $n$. Mémoire d'ingénieur CUST, 2001, p. 6092.
Lieberman S.H., Anderson G.W. - « Use of a direct push video-imaging System for in situ Characterization of subsurface soil environments n. Int. Symp. on imaging applications in geology. Geovision 99, 1999, p. 169-172.

Raschike S.C. - Computer vision for experimental soil micromechanics and soil characterization. Ph. Dissertation, Univ, of Michigan, Ann Arbor, 1996, 256 pages.

SETRA - Remblayage des tranchées et réfection de chaussée. Guide technique (fasc. 1 et 2) 1994. 